\title{
IDIAR: Augmented Reality Dashboards to Supervise Mobile Intervention Studies
}

\author{
Katja Vock \\ Katja.Vock@uni.kn \\ HCI Group, University of Konstanz \\ Germany
}

\author{
Sebastian Hubenschmid \\ Sebastian.Hubenschmid@uni.kn \\ HCI Group, University of Konstanz \\ Germany
}

\author{
Johannes Zagermann \\ Johannes.Zagermann@uni.kn \\ HCI Group, University of Konstanz \\ Germany
}

\author{
Simon Butscher \\ Simon.Butscher@uni.kn \\ HCI Group, University of Konstanz \\ Germany \\ Harald Reiterer \\ Harald.Reiterer@uni.kn \\ HCI Group, University of Konstanz \\ Germany
}

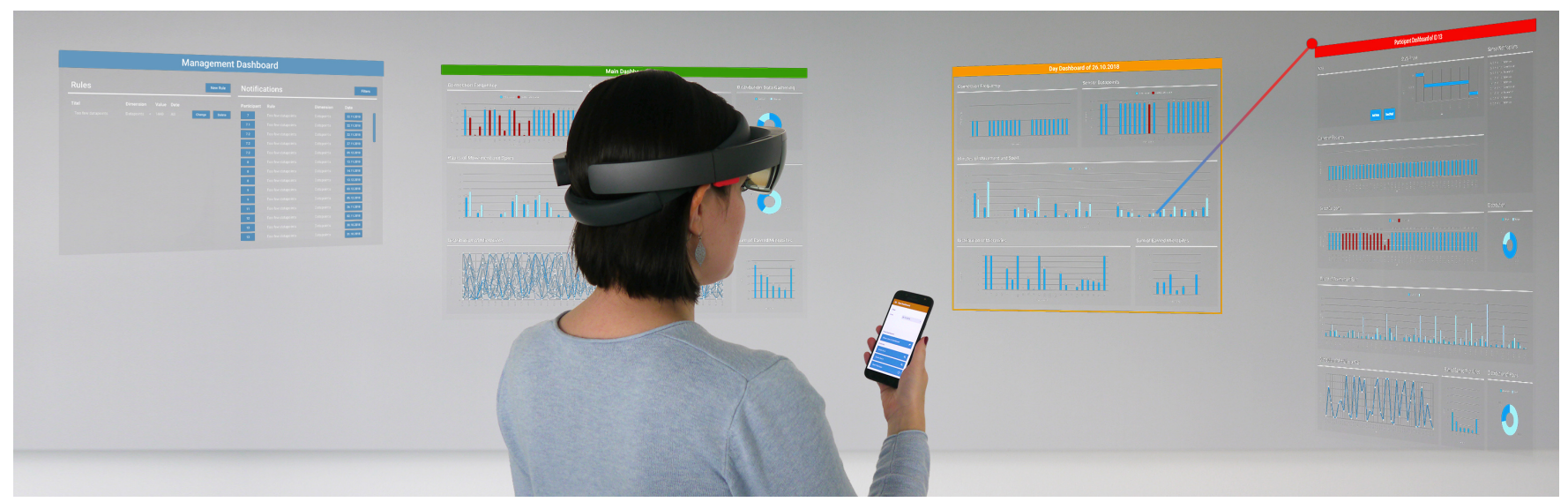

Figure 1: Interactive dashboards in augmented reality (IDIAR) visualize data from an ongoing mobile intervention study, enabling researchers to quickly identify irregularities. Four different dashboards provide users with both general and detailed information about an ongoing mobile intervention study. A multimodal interaction approach combining smartphone-based touch, head gaze, and voice input allows for familiar operation.

\begin{abstract}
Mobile intervention studies employ mobile devices to observe participants' behavior change over several weeks. Researchers regularly monitor high-dimensional data streams to ensure data quality and prevent data loss (e.g., missing engagement or malfunctions). The multitude of problem sources hampers possible automated detection of such irregularities - providing a use case for interactive dashboards. With the advent of untethered head-mounted AR devices, these dashboards can be placed anywhere in the user's physical environment, leveraging the available space and allowing for flexible information arrangement and natural navigation. In this work, we present the user-centered design and the evaluation of IDIAR: Interactive Dashboards in $A R$, combining a headmounted display with the familiar interaction of a smartphone. A
\end{abstract}

This work is licensed under a Creative Commons Attribution International 4.0 License.

MuC '21, September 5-8, 2021, Ingolstadt, Germany

(c) 2021 Copyright held by the owner/author(s).

ACM ISBN 978-1-4503-8645-6/21/09.

https://doi.org/10.1145/3473856.3473876 user study with 15 domain experts for mobile intervention studies shows that participants appreciated the multimodal interaction approach. Based on our findings, we provide implications for research and design of interactive dashboards in AR.

\section{CCS CONCEPTS}

- Human-centered computing $\rightarrow$ Mixed / augmented reality; Interaction techniques.

\section{KEYWORDS}

multimodal interaction, head-mounted displays, mobile devices, evaluation

\section{ACM Reference Format:}

Katja Vock, Sebastian Hubenschmid, Johannes Zagermann, Simon Butscher, and Harald Reiterer. 2021. IDIAR: Augmented Reality Dashboards to Supervise Mobile Intervention Studies. In Mensch und Computer 2021 (MuC '21), September 5-8, 2021, Ingolstadt, Germany. ACM, New York, NY, USA, 12 pages. https://doi.org/10.1145/3473856.3473876 


\section{INTRODUCTION}

The past years have seen a rising interest in keeping track of one's individual physical health using smartphone apps and activity trackers [64]. Researchers in the field of mobile health (mHealth) investigate the efficacy of such apps and devices e.g., by studying their usage and influence on behavior in so called mobile intervention studies that run up to several weeks. During the procedure of these studies, there is a multitude of irregularities that can occur, such as missing engagement of participants (e.g., participant forgets to fill out a daily questionnaire) or malfunction of measuring instruments (e.g., empty battery or Bluetooth connectivity issues). Yet, the complex nature of the data and the multitude and unpredictability of potential problem sources make an automated detection of irregularities difficult (e.g., from lost or broken hardware that needs to be replaced to software-related connectivity issues that can be resolved by rebooting), especially as each study is tailored to answer their individual research questions [53]. Researchers, therefore, have to stay in contact with their participants and regularly monitor the collected high-dimensional data streams during run time of the study to ensure data quality and prevent data loss commonly associated with mHealth data [72]. This monitoring allows for a quick intervention to prevent (i.e., send reminders) or resolve (i.e., replace measuring instruments) irregularities. To this end, interactive dashboards can leverage human capabilities for the identification of patterns and irregularities [21, 45]. Still, visualizing the large amount of data stemming from high-dimensional data and large cohorts of participants (e.g., 20-40 participants) might raise the need for extra screen space - leading to e.g., multi-monitor setups.

With head-mounted mixed reality devices becoming affordable for consumers, these devices present a viable alternative to such multi-monitor setups: Here, a single device allows to dynamically create numerous dashboards that can be scaled or placed to suit workflows, preferences, and physical surroundings. Unlike multimonitor environments, these digital workspaces are location-independent (i.e., users can have the same setup in their regular office or when working from home), can encourage physical activity due to egocentric navigation [3], and increase data confidentiality (i.e., bystanders cannot glance at displays). Compared to virtual reality, the use of augmented reality (AR) can also retain the user's current work practices, such as interacting with colleagues or writing paper notes [43]. Although current head-mounted AR devices still suffer from hardware limitations (e.g., reduced legibility due to low resolution and small field of view), these limitations will likely be resolved by future hardware iterations. Recent research already shows the benefits of using AR devices for visualizing data [6, 28, 57, 62], especially when paired with mobile devices [30, 35, 50, 55], which can also offset some of the disadvantages commonly associated with AR: While common interaction techniques for head-mounted AR devices such as mid-air gestures can be seen as disadvantageous for certain tasks (e.g., resulting in fatigue or inaccuracy [26, 59]), multimodal interaction [38] e.g., by adding common smartphonebased touch interaction $[8,9,28,30]$ can be seen as a promising alternative.

In this work, we present the user-centered design and explorative evaluation of IDIAR: Interactive Dashboards in Augmented Reality for the supervision of mobile intervention studies (see Figure 1). As there is - to the best of our knowledge - no research investigating dashboards, neither in mixed reality nor in study supervision scenarios, our contribution is twofold: (1) Based on the requirements from a focus group with domain experts, we contribute the design of a multimodal interaction approach for interactive dashboards combining smartphone-based touch, voice, and head gaze interaction. (2) The findings of a user study with 15 domain experts in mobile intervention studies. Being an unexplored, yet timely topic, we intentionally focus on qualitative measurements that allow for a rich understanding of how the domain experts (a) use the multimodal interaction techniques, (b) incorporate the cross-device combination of a head-mounted display with a smartphone into their workflow, (c) their user experiences, and (d) the applicability of the IDIAR prototype for everyday professional activities. Based on this, we provide implications for future research and design of dashboards in AR.

\section{RELATED WORK}

We investigate the following three areas to inform the design of our prototype: (1) Mixed reality visualizations, (2) multimodal interaction for data visualizations, and (3) use of cross-device interaction in mixed reality.

\subsection{Mixed Reality Visualizations}

While there is an increasing body of work investigating the benefits of visualizations in mixed reality environments (e.g., [32,69]), the focus often lies on understanding and interacting with 3D visualizations. Yet, research on 2D information spaces in immersive 3D environments shows that 2D spaces are less cluttered [13], more accurate in selection tasks [14], and suitable for the display of simplified information [18]. Recent research also suggests that 2D AR visualizations can be employed in visually challenging environments [62]. In this context, Ren et al. [58] created a prototype for authoring both 2D and 3D visualizations in immersive environments. Furthermore, a recent study by Kraus et al. [31] shows that $2 \mathrm{D}$ visualizations can still outperform their 3D counterpart for overview tasks in virtual reality. Experts often rely on 2D overview visualizations represented as at-a-glance overviews in information dashboards [21], for example in medical [15, 67, 77] or mHealth [19] domains. Yet, to the best of our knowledge, there is no research investigating dashboards in either mixed reality or study supervision scenarios.

\subsection{Multimodal Interaction for Data Visualizations}

Decades of research have demonstrated the benefits of multimodal interaction, such as better accessibility [48], increased flexibility [37], more natural interaction [17], and better task performance [49]. It also allows for more reliability, as users may switch to a more suitable input modality for a given task [47]. Reeves et al. [54] provide general guidelines when designing multimodal interfaces. Recent research has also investigated the use of touch interaction with several other input modalities, such as natural language 
(e.g., [61, 66]), pen (e.g., [65]), proxemic interaction (e.g., [33]), tangible interaction (e.g., [2]), or within immersive environments solutions (e.g., $[10,35,57])$. Especially gaze interaction can be a useful input modality in this scenario, as users have to wear a head-mounted display (HMD) that provides head gaze data without further instrumentation: Here, Pfeuffer et al. show the benefits of combining gaze for selection with touch interaction on a touch-enabled display [51], as well as gaze and freehand gestures in virtual reality [52]. Rivu et al. [60] show how a gaze-enabled AR application can be used to provide beneficial on-demand information, but also easily overwhelm the user. Furthermore, recent work by Pfeuffer et al. [50] provides a design space for integrating such gaze interaction in AR environments (e.g., when switching between real and virtual content, or switching between different tasks).

\subsection{Use of Cross-Device Interaction in Mixed Reality}

The area of cross-device interaction is a popular topic in humancomputer interaction research. Only recently, Brudy et al. [5] provided a comprehensive overview of the domain of cross-device interaction - analyzing a huge variety of topics such as interaction techniques or future key challenges. Their cross-device taxonomy [5] reveals that a major part of this research is in the context of 2D devices: Here, recent research has investigated the usage of tablets (e.g., [75]), smartphones (e.g., [34]), or smartwatches [27]. However, with the advent of consumer mixed reality devices, research has begun to include 3D-capable devices such as HMDs into this stream of research.

In this context, researchers have explored desktops (e.g., [40, 56]), smartphones (e.g., [12, 39, 44]), smartwatches (e.g., [12, 25, 71]), pens (e.g., $[16,70])$, tablets (e.g., $[1,28,68])$, and tabletops (e.g., $[9,24])$ for interacting with $3 \mathrm{D}$ content in mixed reality environments. More specific to this work, Büschel et al. [7] demonstrated that using smartphone-based interaction for navigating 3D data spaces in AR outperforms mid-air gestures and voice input. Building on this, Zhu and Grossman [76] demonstrated the usefulness of smartphones for directly interacting with 3D content in AR, while Grubert et al. [22] have shown that a cross-device interaction approach using AR HMDs and smartphones can outperform a single device. Similarly, Reichherzer et al. [55] created a framework for designing and evaluating such hybrid interfaces that combine AR HMDs with a smartphone. In addition, Knierim et al. [30] showed that smartphone-based interaction for manipulating is both faster and more accurate than mid-air gestures.

\section{SUPERVISION OF MOBILE INTERVENTION STUDIES}

Mobile intervention studies are associated with high efforts for data collection. To prevent these efforts from being in vain, it is important to constantly monitor the quality of the collected data. We consider this as a suitable scenario for investigating the potential of a multimodal interaction approach in an AR environment.

Throughout the paper, we refer to real-world mobile intervention studies that were conducted by an international research consortium consisting of health psychologists, sports psychologists, and human-computer interaction researchers. The studies used the method of ambulatory assessment (comparable to in-the-wild studies) to measure physical activity in everyday life with the aim to investigate how technology can improve mental and physical health. Participants wear movement sensors to track the steps and the activity level per minute. Besides, a custom smartphone app collects additional data via questionnaires. Per cohort, 20 to 40 participants use the tracking tools in a sequence of 5 to 7 weeks. Overall, data from more than thousand participants were collected, whereas, $10-20 \%$ of the data did not pass the retrospective compliance check due to too low data quality (e.g., missing data caused by sensor failure or low participant engagement).

To understand how the supervision of the data collection can improve the data quality, we conducted a focus group interview with five domain experts (psychologists) from the research consortium. Topics included current workflows (including tools), critical situations of current practices, communication activities across the team and with participants, and ideas for improvement. The session was audio-recorded and lasted approximately one hour.

The domain experts mentioned two major reasons to supervise the data collection during mobile intervention studies: technical verification and data plausibility. During the study, participants sometimes face technical or operational problems with the tracking apparatus: The Bluetooth connection between smartphone and the wearable movement sensor can stop working, or participants might forget to track activities or charge the devices. Additionally, plausibility checks are needed if the tracked movement data is beyond the ordinary participant-specific range. These plausibility checks require a root cause analysis to be able to differentiate between atypical but still correctly tracked participant behavior and problems with the measuring tools or the participant's engagement. Consequently, these regular checks and root cause analyses help to identify discrepancies, review them, and, if necessary, contact participants during run time of the study to ensure the data quality and therefore the validity of the results. The domain experts further reported that the complexity, the multitude, and the unpredictability of the problems they experienced with the data collection makes it difficult to provide a comprehensive definition of possible irregularities in advance. Thus, not all problems can be detected automatically, which is why manual monitoring of the data collection is required. The manual monitoring is currently done by looking at spreadsheets holding the raw data. This non-visual approach makes it hard to identify participant-specific patterns and suspicious or missing data points.

To overcome the limitations of the current approach, we propose an interactive dashboard for the supervision of the data collection during mobile intervention studies. Dashboards can provide visual access to the data and therefore facilitate the detection of patterns $[21,45]$. According to the focus group interviews, the requirements that have to be fulfilled can be summarized as follows:

R1-Overview: An at-a-glance overview of the study data can help to identify areas, which require a more detailed analysis.

R2 - Filters: Filters allow for a fast drill-down to assess time periods and individual participants and are required for the identification of root causes. 
R3 - Rules: Using rules, defining data-driven thresholds can help to automatically identify anomalies or irregularities in the data.

R4 - Notes: Being a team effort, note-taking (e.g., linked to participants) can help to communicate insights, issues, or events.

R5 - Communication: A direct communication channel to participants can allow for real-time feedback (e.g., in case of a hardware failure).

While any interactive dashboard solution (e.g., Tableau Dashboard) on single or multi-monitor workplaces could simplify, ease, and better support the current supervision task workflow, we see this as a chance for investigating the benefits and limitations of AR with regard to the use of interactive dashboards. This allows us to gain insights in the use of yet unfamiliar technology, raise questions for future research and design, and explore the feasibility of such a solution.

Furthermore, prior work shows that representing dashboards as 2D information spaces in immersive 3D environments seems to be a promising approach $[18,31]$ to avoid more cluttered and complex 3D visualizations [13]. Dashboards are especially well-suited for providing an overview over a large amount of data (R1), but can also show other information (e.g., rules (R3) and notes (R4)). To fulfil the diverse interaction requirements, we employ a combination of an immersive AR HMD and a smartphone: This combination is not only useful for interacting with 3D content (R1) [30, 76], but also offers a familiar operation when interacting with $2 \mathrm{D}$ content $(\mathrm{R} 2$, R3) or text input (R4, R5) [9, 28]. With this combination, we can also leverage the benefits of multimodal interaction: A multimodal interaction approach can be especially useful when specifying filters (R2), creating rules (R3) and notes (R4), or communicating with study participants (R5), as users can choose the most appropriate input modality for a given task. Especially for text input (R4, R5), the use of touch (i.e., through smartphones or tablets) as additional input modality can provide a familiar input modality [20] and enrich interaction with large visualizations [11,29], while gaze input can be beneficial for selecting information in the overview (R1) [50, 52].

\section{IDIAR - INTERACTIVE DASHBOARD IN AUGMENTED REALITY}

Based on the requirements identified in the focus group and the insights gained from prior work, we designed IDIAR for mHealth researchers to help them in the supervision of mobile intervention studies. IDIAR employs dashboards in AR for an interactive observation of ongoing studies, enabling researchers to track participants' behavior or directly contact participants in case of irregularities. The following sections explain the employed dashboards, our multimodal interaction concept, and the technical setting.

\subsection{Dashboards}

IDIAR uses four dashboards to provide users with information (see Figure 1): A main dashboard (see Figure 2) displays a summarized view over the ongoing study; a day dashboard offers information about the currently selected day; a participant dashboard provides detailed information about a selected participant; and a management dashboard allows users to create or manage detection rules

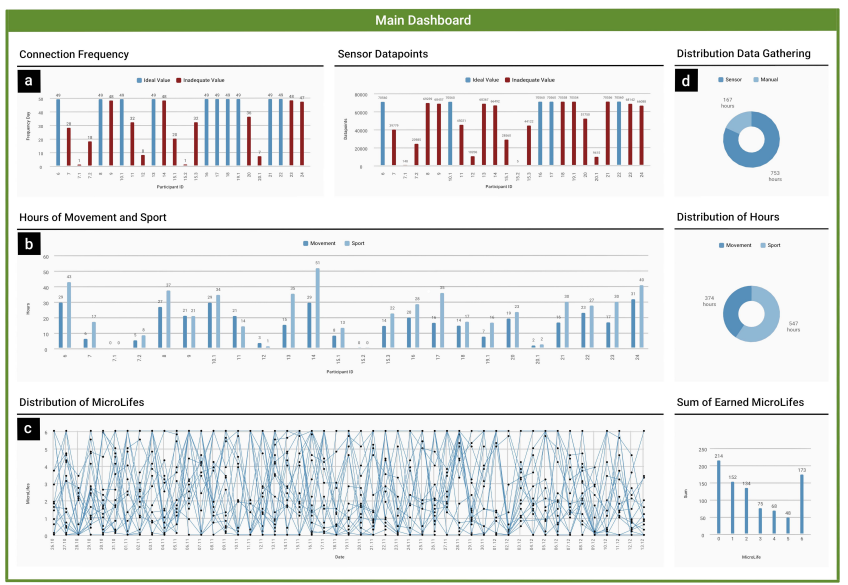

Figure 2: The main dashboard provides a summary of important study metrics: Bar charts show (a) the connection frequency of external sensors and (b) the participants' amount of physical activity; (c) a line chart depicts the trend of an aggregated daily activity value for each participant; and (d) donut charts show general study metrics (e.g., aggregated levels of physical activity). Entries marked in red indicate possible irregularities.

and to see active notifications. Each dashboard employs simple, yet powerful visualizations (i.e., line charts, bar charts, donut charts) to allow users to quickly spot irregularities and determine if an action is necessary (R1). The visualizations also employ visual cues (e.g., colors) based on predefined thresholds (e.g., insufficient sensor values) to highlight possible irregularities that need to be investigated. Following Shneiderman's Visual Information Seeking Mantra [63] ("overview first, zoom and filter, then details-on-demand"), users first start with the main dashboard to get an overview over the current data (R1). The management dashboard allows for a rule-based filtering (e.g., by defining data-related thresholds) (R2, R3), while the day and participant dashboard provide details based on the current selection. We limited IDIAR to these four predefined dashboards with visualizations that mirror existing data structures and control mechanisms. This also allowed for a coherent study setting and reduced complexity. However, future iterations could dynamically add as many dashboards as the users need (e.g., multiple day dashboards to cover one week or multiple participant dashboards to compare them) to leverage the large space afforded by the AR environment. Furthermore, we chose a large dashboard size filling the available field of view to avoid possible legibility issues due to current hardware limitations and provide an at-a-glance overview (R1).

\subsection{Interaction}

With IDIAR, users can interact with the visualizations to investigate data irregularities and communicate with the study participants (e.g., by sending emails). To accommodate these tasks, we adopted a multimodal interaction approach using three interaction techniques that provide a high detection accuracy: (1) head gaze and touch; (2) head gaze and voice; and (3) touch input. The smartphone offers 


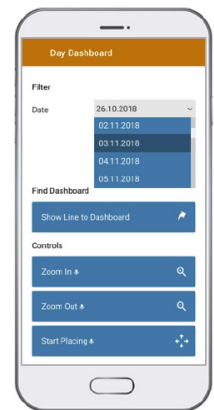

(a)

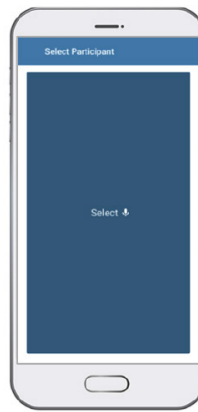

(b)

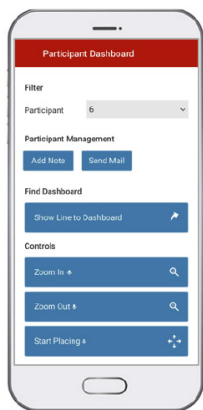

(c)

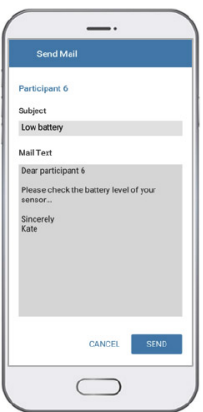

(d)
Figure 3: The smartphone's UI is adapted based on the user's current selection and action: (a) Users can quickly select the appropriate filter option from a drop-down menu. General actions such as placing a dashboard are available as buttons. Buttons marked with a microphone may also be triggered through voice commands. (b) When looking at the data from a specific participant, the smartphone only shows a large button that can be pressed without looking at the smartphone to confirm the selection. (c) When the participant dashboard is selected, the user can interact with available participants by adding a note or sending a notification mail to the participant. (d) When sending a mail, users can leverage the familiar interaction with smartphones to compose a text message.

a context-sensitive UI (see Figure 3) that automatically adapts to the currently selected element. Several actions can also be activated with voice commands, as indicated by a microphone symbol on the smartphone buttons. Furthermore, smartphone interaction provides familiar and efficient text input for messages. Certain actions (i.e., creating a rule, writing an email or annotation) have to be completed on the smartphone; during these actions, the AR view is blocked - informing the user that the task has to be completed on the smartphone. The following sections describe the available features.

4.2.1 Selection. Users can select any element (e.g., dashboard, single data point in a chart, filter rule) via head gaze and confirm the selection with either voice or touch input (see Figure $3 b)$. Being context-sensitive, the smartphone shows a single screen-filling button to allow for selection without the need to look at the phone.

4.2.2 Show Line to Dashboard. Users can show a visual connecting line in AR that guides them to the relevant dashboard in AR (see also Figure 1). This line can be activated manually through a button on the smartphone or will automatically appear when selecting an individual data point, guiding the user to the appropriate dashboard (e.g. when selecting a participant, the user is guided to the participant dashboard that is updated with information about the selected participant).

4.2.3 Filtering. The day and participant dashboards allow users to filter (R2) for specific study days or participants respectively by choosing a value from a drop-down list on the smartphone (see Figure $3 \mathrm{a}$ and $3 \mathrm{c}$ ). In addition, users can filter the result list of the

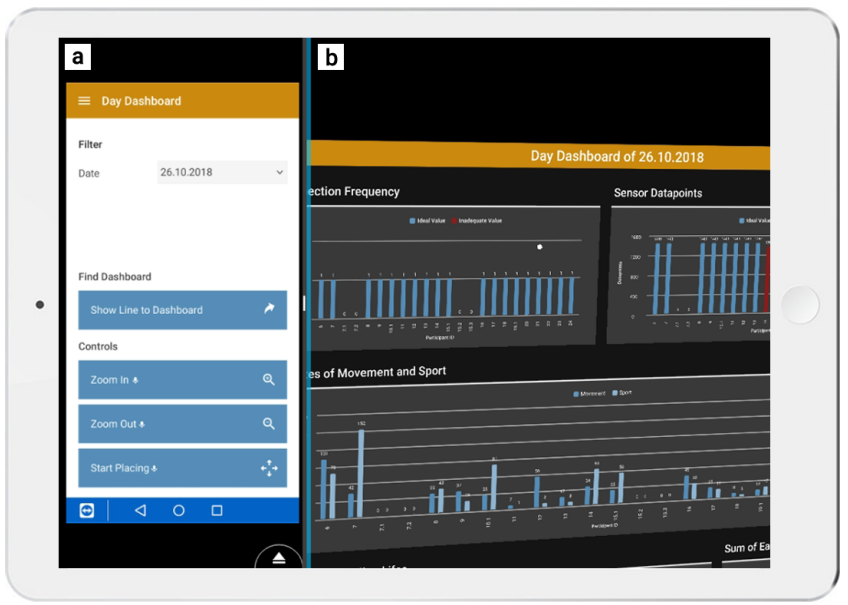

Figure 4: The Observer Mode allows experimenters during user studies to track what the user is currently seeing, both (a) their smartphone and (b) their AR perspective (including current viewing angle). The real-world surroundings are hidden to avoid visual clutter.

management dashboard by participants, rules, data dimensions, and days.

4.2.4 Placing and Zooming. Dashboards can be scaled to zoom using dedicated smartphone buttons or voice commands. They can also be positioned in the physical environment: Here, the position of the dashboard is manipulated by the user's head gaze and confirmed via the smartphone or voice commands. Similar to selection, the smartphone interface is replaced by a large button that can be operated without looking at the device to confirm the placement. This allows users to fully utilize their surrounding space and create their preferred layout.

4.2.5 Establishing Rules. Users can define specific rules (R3) in the management dashboard, which may show a notification if the rule condition is fulfilled. Rules must have a title (e.g., Not enough sensor data), a data dimension (e.g., amount of sensor data), an operator, and a value (e.g., less than 1440), but can also specify a date. The notification list shows an overview of all participants that match a given rule, including their participant ID and the matching rule.

4.2.6 Writing Notes. Notes (R4) can be added using the smartphone through the participant dashboard (see Figure 3c). Users can then enter a text note about current findings (e.g., possible hardware failure) using their smartphone. An overview of existing notes can be found in the participant dashboard.

4.2.7 Sending Emails. Once an irregularity has been discovered, users can contact the study participant through the participant dashboard (R5). Users can inform the participant directly via email, for example, to instruct the participant to charge the sensor's batteries (see Figure 3d). 


\subsection{Technical Setting}

We implemented IDIAR using a Microsoft HoloLens as AR HMD and a Samsung Galaxy J5 smartphone (Android 8.1, resolution: $720 \times 1280$ pixels, display size: $\left.5^{\prime \prime}\right)$ in Unity3D. Communication between the smartphone and HMD uses a client-server architecture, which also allows us to replicate several aspects (e.g., AR perspective) with our Observer Mode (see subsection 5.2 Apparatus).

\section{USER STUDY}

As we were interested in how the IDIAR prototype can live up to real-world requirements, we evaluated it in a user study with domain experts. We focused on qualitative measurements to gain valuable insights how the domain experts use the multimodal interaction techniques (Research Objective 1), incorporate the cross-device combination of a HMD with a smartphone into their workflow (Research Objective 2), their user experiences (Research Objective 3), and the applicability of the IDIAR prototype for everyday professional activities (Research Objective 4).

\subsection{Participants}

Fifteen domain experts (psychologists) (12 female, 3 male) from the research consortium (see Section 2 Supervision of Mobile Intervention Studies) were invited to take part in the user study. Three of them took part in the focus group interviews. Their mean age was 25.6 years ( $\mathrm{SD}=2.69$, aged 23 to 33 ). Three of them were postdocs, four $\mathrm{PhD}$ students, and eight psychology students (working as student researchers). All participants were familiar with using smartphones and six participants stated prior experiences with AR devices (e.g., Microsoft HoloLens). Ten participants have worked with the data of the employed data set before (see subsection Tasks and Data Set).

\subsection{Apparatus}

A walkable 3D volume of approximately $3 \mathrm{~m} \times 2.5 \mathrm{~m} \times 2 \mathrm{~m}$ was allotted as a workspace for participants during the study. This allowed them to move around freely and to naturally navigate within the spatially distributed dashboards. All dashboards were circularly positioned at a distance of two meters from the participant to ensure legibility. The initial state and order of the dashboards is shown in Figure 1. During the study, participants were wearing a Microsoft HoloLens as a head-mounted display and used a Samsung Galaxy $\mathrm{J} 5$ as a hand-held device. All devices were connected to a $5 \mathrm{GHz}$ wireless network to mitigate latency. A desk outside of participant's workspace was used for filling out questionnaires and the final interview. We used two cameras in opposite corners of the room in a bird's-eye view covering the entire workspace to see interactions and working behaviors (see Figure 5). The experimenter used a laptop to control the procedure and a tablet that showed the Observer Mode: When evaluating AR applications or supporting a participant during an AR study, the experimenter does not necessarily see what the participant sees, which can be problematic. Although Microsoft provides an option to see the current Microsoft HoloLens view on a web interface, this slows down the application on the Microsoft HoloLens itself and the transmitted view is delayed. Consequently, we used our client-server architecture to implement an Observer Mode of IDIAR for tablet devices (see Figure 4, cf. [28, 74]). This Observer Mode replicates a view of the participant's AR view, yet

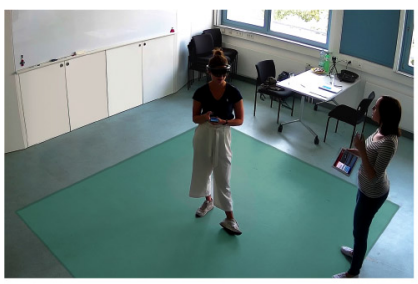

(a)

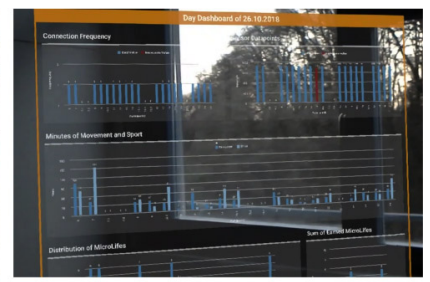

(b)
Figure 5: (a) Setting of the user study with augmented workspace overlay (not visible during the user study). (b) Participant's point of view during the study.

without real-world surroundings to avoid visual clutter. Additionally, the Observer Mode is supplemented with a real-time view of the participant's smartphone (using TeamViewer). The Observer Mode allows experimenters to observe and support participants during a user study without intruding the user experience of AR. We decided to run the Observer Mode on a tablet, as this solution provides a first-person perspective of the participant's point of view without entering their personal space (cf. using a second HMD as an experimenter), which could potentially influence participants' behavior. The Observer Mode used a Samsung Galaxy Tab A tablet (Android 6, resolution: $1024 \times 768$ pixels, display size: 9.7"').

\subsection{Procedure}

First, the experimenter welcomed participants and explained the purpose of the user study, its procedure, and the differences between augmented and virtual reality. After signing the consent form, participants were asked to fill out a questionnaire about demographics, tech-savviness, and prior data set experiences. Then, the experimenter launched IDIAR on the HMD and smartphone and handed them to the participant to start a training phase. During this training phase, the experimenter explained all possible dashboards and interaction techniques and participants were tasked to try all techniques until they felt comfortable using it. After this phase, the experimenter presented the actual tasks with the remark that there are different possible approaches to solve the tasks and that participants can freely choose their preferred interaction technique. Participants were asked to follow a thinking-aloud protocol while completing the tasks. The session was concluded by a user experience questionnaire and a semi-structured interview. Each session lasted about 45 minutes and participants were thanked for their time. We followed all ethical and sanitary guidelines provided by our local university.

\subsection{Tasks and Data Set}

The domain experts provided us with the opportunity to incorporate one of their data sets that was completed a few weeks beforehand in our study prototype. Having the possibility of a real-life data set in combination with domain experts as participants for a user study, we decided to simulate actual work practices as tasks. Therefore, we asked the domain experts during the initial focus group interview for specific daily activities. This allowed us to study participants' work behavior with higher ecological validity. Our tasks included the detection of irregularities (e.g., missing sensor data), 
handling these errors, and informing participants or other research group members. Additionally, another typical activity included the definition of data-based rules to detect, for example, unrealistic movement data per day.

\subsection{Data Collection and Analysis}

We employed several data collection methods: We focused on qualitative insights that were supplemented, e.g., by questionnaires and data logs. We used video recordings for the verification of participants' statements and data logs.

5.5.1 Semi-structured Interview and Thinking-Aloud. We gained qualitative insights into participants' activities and experiences by asking them to follow a thinking-aloud protocol during task completion. We also asked participants during a concluding semistructured interview for comparisons to their regular work activities, subjective preferences for input modalities, and if they could imagine to use such a system in their everyday work. All sessions and the concluding interview were audio and video recorded. We fully transcribed all interviews and participants' statements, which where then qualitatively coded following a thematic analysis approach [4]. We iteratively coded for statements describing the use of interaction techniques or devices, experienced advantages or disadvantages, and references to current work practices. All interviews and statements were coded by one person to ensure consistency.

5.5.2 Questionnaires. Besides a demographic questionnaire that provided information on personal data, experiences with $A R$, and prior knowledge about the data set, we utilized the User Experience Questionnaire (UEQ) [36] to gain comprehensive information on participants' experiences.

5.5.3 Data Logs. We collected logs of participants' interaction with the devices, namely the number of interactions with the smartphone (e.g., button presses) and the usage of voice commands. We did this to see if participants e.g., prefer one of these input modalities in general or for certain subtasks.

\section{FINDINGS AND DISCUSSION}

We report and discuss our findings in relation to our research objectives. We focus on the use of multimodal interaction techniques (Research Objective 1), the cross-device use of head-mounted and hand-held devices (Research Objective 2), the perceived user experience (Research Objective 3), and the applicability of such a system in everyday professional activities (Research Objective 4). Based on our findings, we provide implications for research and design that can help researchers and practitioners to study and design interactive dashboards in AR.

\subsection{Research Objective 1 - Multimodal Interaction Techniques}

Participants were free to choose their preferred interaction technique, either as a single technique or in combination with another one: Head gaze and smartphone-based touch input, head gaze and voice command, and touch input on the smartphone only. During task completion, participants used at least two $(n=10)$ to up to three $(n=5)$ techniques. Based on the concluding interview, there was no tendency towards one of the techniques. However, data logs revealed that participants used the combination of head gaze and touch input for $47 \%$ of all interactions. Touch input only was used for $27 \%$ and the combination of head gaze, and voice commands for $26 \%$ of all interactions. One participant advocated for voice commands: "[...] it is very intuitive ... simply gaze and speak. I think that is the method with the highest speed" - [P11], while another participant reflected critically on voice commands in general: "I never use voice commands and thus I am not used to it. I think its strange to speak into an empty space" - [P5]. Participants liked the immediacy of touch input on the smartphone: "You see at the corner of the eye that the confirmation button appeared [on the smartphone] and then you can directly click on it" - [P13]. Participants' choice of modality was also informed by the current activity at hand: "I used the smartphone only for direct input like writing an email." - [P1].

The multimodal interaction approach allowed participants to select the input modality that fits best and to recover from errors. "I belief that I prefer voice commands because I do not have to use the smartphone. [...] I felt that I was faster with the voice commands." [P1]. If a voice command failed, participants tried again or switched to touch input, which is in line with previous research (e.g., [46]).

Most domain experts $(n=10)$ mentioned difficulties with the head gaze interaction, especially to keep the selection with their head gaze during an interaction with voice commands (cf. Heisenberg effect of spatial interaction [73]): "To hold the [head] gaze still during the voice commands was the most difficult part of the interactions" [P7]. Participants quickly developed different coping mechanisms: (1) Participants zoomed the interface to have larger buttons and bars and (2) avoided voice commands and used touch input on the smartphone to confirm the selection instead.

Due to technical difficulties, three domain experts encountered a lag in the reaction time of voice commands. They were unsure whether a voice command was recognized by the IDIAR prototype or if they should repeat it: "The reaction based on the voice commands did not respond fast enough. Thus, I was unsure if it has worked" - [P2]. In this case, participants missed an additional visual or auditory feedback if a voice command was recognized.

\section{Implications for Research and Design - Multimodal} Interaction Techniques

Participants preferred the combination of head gaze and touch input, liked the immediacy of touch input on a smartphone, and had polarized opinions regarding voice commands. However, all modalities were used complementary (cf. [46]), allowing for preferences and error recovery, while increasing IDIAR's robustness.

$\longrightarrow$ As all provided interaction techniques showed strengths and drawbacks, further research is necessary to (1) improve their usage (e.g., finding a sweet spot for target sizes within a limited field of view), (2) increase their acceptance (e.g., adding a voice command agent to avoid the impression of speaking into the void), and (3) study their usage over time and in the wild (e.g., identifying coping mechanism and authentic usage patterns). 


\subsection{Research Objective 2-Cross-Device Interaction}

Participants handled the simultaneous usage of the HMD and the smartphone without major problems. They described the use of the HMD as a rather implicit activity: "It was unproblematic. You do not use the augmented reality glasses actually. It was no control device" - [P2]. Here, separating the visual representation and control was a design decision, which proved to be a promising approach in previous research (e.g., [29, 33]). In line with this, most domain experts described this distribution as "reasonable" and "pleasant".

Similar to Büschel et al. [7], participants stated that their familiarity with the smartphone was an advantage to control the IDIAR prototype: "I am more used to the smartphone and so I know what I am doing. [...] It would be exhausting to select every letter individually in augmented reality" - [P6].

Only one participant had difficulties with the combination of two devices and the affordances of their usage: "Sometimes I was not sure if I have to do something in the dashboards or on the smartphone. Some parts were only possible to do on the smartphone, like to create rules" - [P14]. Also, the IDIAR prototype required participants to occasionally switch their focus from the AR visualization to the content of the smartphone or vice versa. While previous research described this focus switch rather critically [23], only two participants described this as "cumbersome" during the concluding interview.

\section{Implications for Research and Design - Cross-Device Interaction \\ Separating visual representation and control to two devices was appreciated by our participants (cf. [29, 33]), allowing for familiar interaction in an AR environment [7], while utilizing individual benefits of each device [20]. \\ $\longrightarrow$ Further research is necessary to increase the affordances of each device's capabilities. Here, sticking to the concept of separating the visual representation and its control could be further extended by e.g. allowing for eyes-free text entry on the mobile device while showing the text in AR (cf. [41]). This could in turn reduce switching costs. Also, comparing alternative device combinations might lead to interesting in- sights: While larger mobile devices (i.e., tablets) could allow to integrate the additional modality of pen input for notes on the mobile device, smaller mobile devices (i.e., smart- watches) could lead to new interaction patterns (cf. [27]).}

\subsection{Research Objective 3 - User Experience}

The UEQ [36] was used to evaluate the user experience of the IDIAR prototype. Having a possible range of scores from -3 to 3 , all scales were rated positive. The attractiveness scale was rated as good (mean $=1.99, \mathrm{SD}=0.21)$ and the two hedonic scales - stimulation and novelty - show remarkable good means of $2.43(\mathrm{SD}=0.31)$ and $2.47(\mathrm{SD}=0.41)$.

These scores show participants' high interest in the IDIAR prototype and that they were motivated to use it. One participant stated: "My first impression of IDIAR was really good. Very unexpected that it is possible to have it located entirely over the room with the various dashboards" - [P4]. The pragmatic scales perspicuity, efficiency, and dependability have mean values of $1.58(\mathrm{SD}=0.27), 1.35$ (SD $=0.56)$, and $1.18(\mathrm{SD}=0.37)$, respectively. Theses values might be related to participants' prior knowledge, their expectations, as well as the novelty of the prototype itself.

Participant's prior knowledge and with this the learning curve of the IDIAR prototype was mentioned in the interview: "Disadvantages? You have to get used to it [IDIAR] a bit in order to know where what is located and how to use it" - [P4]. P6 added "Even though it is something new, you understand relatively easily how it works." When asked about the strengths and weaknesses of the IDIAR prototype, some domain experts $(n=5)$ stated that the HMD was uncomfortable to wear: "[The weakness of IDIAR was] definitely the [augmented reality] glasses. It started to pinch and as a wearer of glasses, it was hard to put it on comfortable." - [P5]. Four participants emphasized how beneficial the IDIAR prototype could be for their personal health, as it promotes working while standing or moving: "It [moving around] is also a good aspect for health management in companies. You get simply the chance to stand up and move around." $-[\mathrm{P} 11]$.

\section{Implications for Research and Design - User Experi- ence}

Subjective ratings and the concluding interview showed a high user experience of the IDIAR prototype, highlighting strengths (e.g., attractiveness) and weaknesses (e.g., comfort) for using the prototype and wearing the HMD for the duration of a study session.

$\longrightarrow$ Longitudinal research is necessary to (1) investigate to what extend the mentioned strengths can be attributed to a novelty effect, (2) identify a learning curve, and (3) study which type of HMD is suitable for prolonged use.

\subsection{Research Objective 4-Applicability}

Thirteen participants liked the dashboards: "The advantage is that you have everything at a glance... but also very detailed information like sensor data or movement data" - [P4]. The division into dedicated dashboards with different levels of granularity and purposes was also appreciated by 12 participants: "I think it was refreshing to have different dashboards because I had the feeling you see a lot. They support you in getting the answers to different questions you may have. For example, I only want to see extreme outliers or to have a view of one day or one participant" - [P6]. Participants were familiar with the data representations: "The visualizations were easily understandable. I took a look and knew what I saw" - [P2]. Further, participants also compared the IDIAR prototype to their current work practices: "When I imagine to analyze this amount of data with a computer that will not work, because also with 5 screens I would continuously have to click around and the comparison [of data points] would not be that good" - [P3] and P10 added "It was exciting and thrilling because you can move around [in the data]" - [P10], reflecting on the immersive analytics approach. Participants liked the re-positioning of dashboards in space, which was described as "To move your whole dashboard, you only have to look somewhere. It was totally easy" - [P7]. The possibility to communicate with a study 
participant through the interface was valued by the domain experts: "It would be a huge advantage if I can contact the participants directly over the system. Therefore I can save a lot of intermediate stages and also time” - [P1]. Also, setting up rules (e.g., for certain thresholds) was seen as beneficial for the workflow: "It is a cool thing because with the different rules, I had the feeling that it was very fast and intuitive" - [P6]. Some domain experts missed a transfer to their conventional work environment (i.e., a printout or a screenshot), or to other applications (e.g., for statistical analyses), and asked for collaborative features (e.g., to discuss findings with peers). Overall, eleven of fifteen domain experts could imagine using IDIAR in the future: "If there is a possibility to use it, by all means. I think it would be exciting to look at the data this way" - [P4]. Other participants rated the lack of space and equipment as a challenge to use IDIAR in their everyday professional life. However, these issues might vanish by decreasing hardware costs and increasing hardware fidelity, which could allow for adjustable dashboards that fit to the physical environment and workflow without endangering information legibility.

\section{Implications for Research and Design - Applicability} Participants appreciated following aspects of IDIAR: (1) Using interactive dashboards were seen as a major improvement to their current work practices, as they allow for at-aglance overviews on different levels of granularity. (2) Visualizing these dashboards in AR provided benefits such as a certain degree of immersion, allowing them to "move around [in the data]". Further, they mentioned several drawbacks that can be seen as basis for future work:

$\longrightarrow$ Since we focused on a qualitative evaluation, we did not compare IDIAR's usage with multi-monitor setups. However, future work could compare AR environments with equivalent (multi)-monitor setups to study differences regarding readability, awareness, and quality of work.

$\longrightarrow$ We chose a simple yet comparable arrangement of dashboards for our study. Future work could investigate dashboard configurations (e.g., by attaching them to physical objects) that are efficient in their use of space while being effective regarding spatial memory.

$\longrightarrow$ Future research could investigate collaboration possibilities: (1) Providing each collaborator with an IDIAR set (i.e., HMD and smartphone) could allow for symmetric collaboration, (2) Borrowing the ideas of the Observer Mode could result in the establishment of different roles, and (3) Combining AR environments with traditional desktop settings could allow for individual and collaborative transitions (co-located and remote).

\section{LIMITATIONS \& FUTURE WORK}

Our user study with the IDIAR prototype and domain experts was intentionally focused on qualitative measurements. This allowed for a rich understanding of our participants' experiences, their handling of the device combination, and how the IDIAR prototype could live up to real-world requirements. Although we included quantitative metrics (i.e., data logs) e.g., to find differences in the number of times an interaction technique was used, future work could further investigate the effectiveness and efficiency of the techniques. Measures of task completion time, error, and task load in a comparative evaluation could lead to a more nuanced description of how participants use multimodal interaction techniques, compare the use of a smartphone against mid-air gestures when interacting with dashboards (cf. [30]), or measure the effects of prolonged use of a HMD (e.g. by comparing the combination of smartphone with HMD against a smartphone with multi-display setup).

Similarly, despite recent advances, the hardware is still limited with regard to field of view, legibility, and application performance. This also influenced our choice of input modalities, as e.g. eyetracking was not readily available. Although we designed IDIAR with these limitations in mind, an extended interaction concept could investigate further interaction possibilities with e.g. eyetracking (cf. [50], or investigate the combination of smartphone interaction and mid-air gestures (cf. [42]).

Although our work investigated the specific use case for mobile intervention studies - targeted to a specific user group, some findings might also be valid for other domains that focus on longitudinal studies. Future work could investigate other scenarios and domains that require the supervision of longitudinal data collection (e.g., diary studies). Besides that, a longitudinal study of a system like IDIAR itself could reveal additional requirements, problems, or usage patterns of multimodal interaction techniques with HMDs and smartphones.

\section{CONCLUSION}

With this work, we contribute to the unexplored use case of supervising mobile intervention studies with interactive AR dashboards. Based on a focus group with domain experts, we identified current issues when supervising mobile intervention studies. We contribute IDIAR, a prototype that addresses these issues by providing interactive dashboards in immersive AR, allowing researchers to quickly identify irregularities within ongoing studies and intervene by sending messages to participants. The AR environment provides a larger and more flexible workspace in terms of scale and organization of dashboards, while still keeping the users' current work practices. IDIAR leverages a multimodal interaction approach combining smartphone-based touch, head gaze, and voice input, thus allowing users to choose their preferred interaction modalities. We studied IDIAR in a user study with 15 domain experts, showing that the multimodal interaction helps in error recovery, while the familiar smartphone interaction allowed participants to understand the system quickly and provided a sense of immediacy, which was especially useful for writing text. Participants also appreciated the use of space afforded by both the AR environment and the organization of dashboards. Based on these findings, we provide implications for research and design that can help researchers and practitioners to study and design future interactive dashboards in AR.

\section{ACKNOWLEDGMENTS}

This research was funded by the Deutsche Forschungsgemeinschaft (DFG, German Research Foundation) - Project-ID 251654672 TRR 161 (Project C01) and SMARTACT (BMBF, Grant 01EL1820A). 


\section{REFERENCES}

[1] Rahul Arora, Rubaiat Habib Kazi, Tovi Grossman, George Fitzmaurice, and Karan Singh. 2018. SymbiosisSketch: Combining 2D \& 3D Sketching for Designing Detailed 3D Objects in Situ. In Proceedings of the 2018 CHI Conference on Human Factors in Computing Systems - CHI '18 (CHI '18). ACM Press, Montreal QC, Canada, 1-15. https://doi.org/10.1145/3173574.3173759

[2] Lonni Besançon, Mickael Sereno, Lingyun Yu, Mehdi Ammi, and Tobias Isenberg. 2019. Hybrid Touch/Tangible Spatial 3D Data Selection. Computer Graphics Forum 38, 3 (jun 2019), 553-567. https://doi.org/10.1111/cgf.13710_eprint: https://onlinelibrary.wiley.com/doi/pdf/10.1111/cgf.13710.

[3] Mark Billinghurst, Adrian Clark, and Gun Lee. 2015. A Survey of Augmented Reality. Foundations and Trends ${ }^{\circledR}$ in Human-Computer Interaction 8, 2-3 (2015), 73-272. https://doi.org/10.1561/1100000049

[4] R.E. Boyatzis. 1998. Transforming Qualitative Information: Thematic Analysis and Code Development. SAGE Publications, Thousand Oaks, CA. https://books google.ch/books?id=_rfClWRhIKAC

[5] Frederik Brudy, Christian Holz, Roman Rädle, Chi-Jui Wu, Steven Houben, Clemens Nylandsted Klokmose, and Nicolai Marquardt. 2019. Cross-Device Taxonomy: Survey, Opportunities and Challenges of Interactions Spanning Across Multiple Devices. In Proceedings of the 2019 CHI Conference on Human Factors in Computing Systems - CHI '19. ACM Press, Glasgow, Scotland Uk, 1-28. https://doi.org/10.1145/3290605.3300792

[6] Wolfgang Büschel, Anke Lehmann, and Raimund Dachselt. 2021. MIRIA: A Mixed Reality Toolkit for the In-Situ Visualization and Analysis of Spatio-Temporal Interaction Data. In Proceedings of the 2021 CHI Conference on Human Factors in Computing Systems (CHI '21). ACM, New York, NY, USA, 1-15. https://doi.org/ $10.1145 / 3411764.3445651$

[7] Wolfgang Büschel, Annett Mitschick, Thomas Meyer, and Raimund Dachselt. 2019. Investigating Smartphone-Based Pan and Zoom in 3D Data Spaces in Augmented Reality. In Proceedings of the 21st International Conference on HumanComputer Interaction with Mobile Devices and Services - MobileHCI '19. ACM Press, Taipei, Taiwan, 1-13. https://doi.org/10.1145/3338286.3340113

[8] Wolfgang Büschel, Patrick Reipschläger, Ricardo Langner, and Raimund Dachselt 2017. Investigating the Use of Spatial Interaction for 3D Data Visualization on Mobile Devices. In Proceedings of the Interactive Surfaces and Spaces on ZZZ - ISS '17 (ISS '17). ACM Press, Brighton, United Kingdom, 62-71. https://doi.org/10. $1145 / 3132272.3134125$

[9] Simon Butscher, Sebastian Hubenschmid, Jens Müller, Johannes Fuchs, and Harald Reiterer. 2018. Clusters, Trends, and Outliers: How Immersive Technologies Can Facilitate the Collaborative Analysis of Multidimensional Data. In Proceedings of the 2018 CHI Conference on Human Factors in Computing Systems - CHI '18. ACM Press, New York, New York, USA, 1-12. https://doi.org/10.1145/3173574.3173664

[10] Marco Cavallo, Mishal Dolakia, Matous Havlena, Kenneth Ocheltree, and Mark Podlaseck. 2019. Immersive Insights: A Hybrid Analytics System for Collaborative Exploratory Data Analysis. In 25th ACM Symposium on Virtual Reality Software and Technology. ACM, Parramatta, NSW, Australia, 1-12. https://doi.org/10. 1145/3359996.3364242

[11] Mohammad Chegini, Keith Andrews, Tobias Schreck, and Alexei Sourin. 2019 Multiple Linked-View Exploration on Large Displays Facilitated by a Secondary Handheld Device. In International Workshop on Advanced Image Technology (IWAIT) 2019, Phooi Yee Lau, Kazuya Hayase, Qian Kemao, Wen-Nung Lie, YungLyul Lee, Sanun Srisuk, and Lu Yu (Eds.). SPIE, Singapore, Singapore, 150. https //doi.org/10.1117/12.2521648

[12] Yuan Chen, Keiko Katsuragawa, and Edward Lank. 2020. Understanding Viewport- and World-Based Pointing with Everyday Smart Devices in Immersive Augmented Reality. In Proceedings of the 2020 CHI Conference on Human Factors in Computing Systems (CHI '20). Association for Computing Machinery, Honolulu, HI, USA, 1-13. https://doi.org/10.1145/3313831.3376592

[13] Andy Cockburn and Bruce McKenzie. 2002. Evaluating the Effectiveness of Spatial Memory in 2D and 3D Physical and Virtual Environments. In Proceedings of the SIGCHI conference on Human factors in computing systems Changing our world, changing ourselves - CHI '02 (CHI '02). ACM Press, Minneapolis, Minnesota, USA, 203-210. https://doi.org/10.1145/503376.503413

[14] A. Cockburn, P. Quinn, C. Gutwin, G. Ramos, and J. Looser. 2011. Air Pointing Design and Evaluation of Spatial Target Acquisition with and without Visual Feedback. International fournal of Human-Computer Studies 69, 6 (jun 2011), 401-414. https://doi.org/10.1016/j.ijhcs.2011.02.005

[15] Robin De Croon, Joris Klerkx, and Erik Duval. 2015. Design and Evaluation of an Interactive Proof-of-Concept Dashboard for General Practitioners. In 2015 International Conference on Healthcare Informatics. IEEE, 150-159. https://doi org/10.1109/ICHI.2015.25

[16] Tobias Drey, Jan Gugenheimer, Julian Karlbauer, Maximilian Milo, and Enrico Rukzio. 2020. VRSketchIn: Exploring the Design Space of Pen and Tablet Interaction for 3D Sketching in Virtual Reality. In Proceedings of the 2020 CHI Conference on Human Factors in Computing Systems (CHI '20). Association for Computing Machinery, Honolulu, HI, USA, 1-14. https://doi.org/10.1145/3313831.3376628
[17] Bruno Dumas, Denis Lalanne, and Sharon Oviatt. 2009. Multimodal Interfaces: A Survey of Principles, Models and Frameworks. In Lecture Notes in Computer Science. Springer Berlin Heidelberg, Berlin, Heidelberg, 3-26. https://doi.org/10. 1007/978-3-642-00437-7 1

[18] Barrett Ens, Juan David Hincapié-Ramos, and Pourang Irani. 2014. Ethereal Planes: A Design Framework for 2D Information Space in 3D Mixed Reality Environments. In Proceedings of the 2nd ACM symposium on Spatial user interaction - SUI '14 (SUI '14). ACM Press, Honolulu, Hawaii, USA, 2-12. https: //doi.org/10.1145/2659766.2659769

[19] Dezhi Fang, Fred Hohman, Peter Polack, Hillol Sarker, Minsuk Kahng, Moushumi Sharmin, Mustafa al'Absi, and Duen Horng Chau. 2017. mHealth Visual Discovery Dashboard. In Proceedings of the 2017 ACM International foint Conference on Pervasive and Ubiquitous Computing and Proceedings of the 2017 ACM International Symposium on Wearable Computers (UbiComp '17). ACM, Maui, Hawaii, 237-240. https://doi.org/10.1145/3123024.3123170

[20] Steven Feiner and Ari Shamash. 1991. Hybrid User Interfaces: Breeding Virtually Bigger Interfaces for Physically Smaller Computers. In Proceedings of the 4th annual ACM symposium on User interface software and technology - UIST '91 (UIST '91). ACM Press, Hilton Head, South Carolina, USA, 9-17. https://doi.org/10. $1145 / 120782.120783$

[21] Stephen Few. 2006. Information Dashboard Design: The Effective Visual Communication of Data. O'Reilly, Beijing Cambridge MA.

[22] Jens Grubert, Matthias Heinisch, Aaron Quigley, and Dieter Schmalstieg. 2015. MultiFi: Multi Fidelity Interaction with Displays On and Around the Body. In Proceedings of the 33rd Annual ACM Conference on Human Factors in Computing Systems - CHI '15 (CHI '15). ACM Press, Seoul, Republic of Korea, 3933-3942. https://doi.org/10.1145/2702123.2702331

[23] Jens Grubert, Matthias Kranz, and Aaron Quigley. 2016. Challenges in Mobile Multi-Device Ecosystems. mUX: The fournal of Mobile User Experience 5, 1 (aug 2016), 1-22. https://doi.org/10.1186/s13678-016-0007-y

[24] Martin Hachet, Benoit Benoît Benoit Benoît Bossavit, Aurelie Cohe, Jean-Baptiste de la Riviere, Aurélie Cohé, and Jean-Baptiste de la Rivière. 2011. Toucheo: Multitouch and Stereo Combined in a Seamless Workspace. In Proceedings of the 24th annual ACM symposium on User interface software and technology - UIST '11. ACM Press, New York, NY, USA, 587-592. https://doi.org/10.1145/2047196. 2047273

[25] Steven J. Henderson and Steven Feiner. 2009. Evaluating the Benefits of Augmented Reality for Task Localization in Maintenance of an Armored Personnel Carrier Turret. In 2009 8th IEEE International Symposium on Mixed and Augmented Reality. IEEE, 135-144. https://doi.org/10.1109/ISMAR.2009.5336486

[26] Juan David Hincapié-Ramos, Xiang Guo, Paymahn Moghadasian, and Pourang Irani. 2014. Consumed Endurance: A Metric to Quantify Arm Fatigue of Mid-Air Interactions. In Proceedings of the 32nd annual ACM conference on Human factors in computing systems - CHI '14 (CHI '14). ACM Press, Toronto, Ontario, Canada, 1063-1072. https://doi.org/10.1145/2556288.2557130

[27] Tom Horak, Sriram Karthik Badam, Niklas Elmqvist, and Raimund Dachselt. 2018. When David Meets Goliath: Combining Smartwatches with a Large Vertical Display for Visual Data Exploration. In Proceedings of the 2018 CHI Conference on Human Factors in Computing Systems - CHI '18. ACM Press, New York, New York, USA, 1-13. https://doi.org/10.1145/3173574.3173593

[28] Sebastian Hubenschmid, Johannes Zagermann, Simon Butscher, and Harald Reiterer. 2021. STREAM: Exploring the Combination of Spatially-Aware Tablets with Augmented Reality Head-Mounted Displays for Immersive Analytics. In Proceedings of the 2021 CHI Conference on Human Factors in Computing Systems. ACM, New York, NY, USA, Article 469, 14 pages. https://doi.org/10.1145/3411764. 3445298

[29] U. Kister, K. Klamka, C. Tominski, and R. Dachselt. 2017. GraSp: Combining Spatially-Aware Mobile Devices and a Display Wall for Graph Visualization and Interaction. Computer Graphics Forum 36, 3 (jun 2017), 503-514. https: //doi.org/10.1111/cgf.13206

[30] Pascal Knierim, Dimitri Hein, Albrecht Schmidt, and Thomas Kosch. 2021. The SmARtphone Controller: Leveraging Smartphones as Input and Output Modality for Improved Interaction within Mobile Augmented Reality Environments. i-com 20, 1 (apr 2021), 49-61. https://doi.org/10.1515/icom-2021-0003

[31] Matthias Kraus, Katrin Angerbauer, Juri Buchmüller, Daniel Schweitzer, Daniel A. Keim, Michael Sedlmair, and Johannes Fuchs. 2020. Assessing 2D and 3D Heatmaps for Comparative Analysis: An Empirical Study. In Proceedings of the 2020 CHI Conference on Human Factors in Computing Systems (CHI'20). Association for Computing Machinery, Honolulu, HI, USA, 1-14. https://doi.org/10. $1145 / 3313831.3376675$

[32] M. Kraus, N. Weiler, D. Oelke, J. Kehrer, D. A. Keim, and J. Fuchs. 2020. The Impact of Immersion on Cluster Identification Tasks. IEEE Transactions on Visualization and Computer Graphics 26, 1 (jan 2020), 525-535. https://doi.org/10.1109/TVCG. 2019.2934395

[33] Ricardo Langner, Tom Horak, and Raimund Dachselt. 2018. VisTiles: Coordinating and Combining Co-Located Mobile Devices for Visual Data Exploration. IEEE Transactions on Visualization and Computer Graphics 24, 1 (jan 2018), 626-636. https://doi.org/10.1109/TVCG.2017.2744019 
[34] Ricardo Langner, Ulrike Kister, and Raimund Dachselt. 2019. Multiple Coordinated Views at Large Displays for Multiple Users: Empirical Findings on User Behavior, Movements, and Distances. IEEE Transactions on Visualization and Computer Graphics 25, 1 (jan 2019), 608-618. https://doi.org/10.1109/TVCG.2018. 2865235

[35] Ricardo Langner, Marc Satkowski, Wolfgang Büschel, and Raimund Dachselt. 2021. MARVIS: Combining Mobile Devices and Augmented Reality for Visual Data Analysis. In Proceedings of the 2021 CHI Conference on Human Factors in Computing Systems (CHI '21). ACM, New York, NY, USA, 1-17. https://doi.org/ $10.1145 / 3411764.3445593$

[36] Bettina Laugwitz, Theo Held, and Martin Schrepp. 2008. Construction and Evaluation of a User Experience Questionnaire. In Lecture Notes in Computer Science, Andreas Holzinger (Ed.). Springer Berlin Heidelberg, Berlin, Heidelberg, 63-76. https://doi.org/10.1007/978-3-540-89350-9_6

[37] Joseph J. LaViola, Ernst Kruijff, Ryan P. McMahan, Doug A. Bowman, and Ivan Poupyrev. 2017. 3D User Interfaces: Theory and Practice (second edition ed.). Pearson Addison Wesley Prof, Boston.

[38] Bongshin Lee, Eun Kyoung Choe, Petra Isenberg, Kim Marriott, and John Stasko. 2020. Reaching Broader Audiences With Data Visualization. IEEE Computer Graphics and Applications 40, 2 (mar 2020), 82-90. https://doi.org/10.1109/MCG. 2020.2968244

[39] Chi-Jung Lee and Hung-Kuo Chu. 2018. Dual-MR: Interaction with Mixed Reality Using Smartphones. In Proceedings of the 24th ACM Symposium on Virtual Reality Software and Technology (VRST '18). ACM, Tokyo, Japan, 1-2. https://doi.org/10. $1145 / 3281505.3281618$

[40] Jinha Lee, Alex Olwal, Hiroshi Ishii, and Cati Boulanger. 2013. SpaceTop: Integrating 2D and Spatial 3D Interactions in a See-through Desktop Environment. In Proceedings of the SIGCHI Conference on Human Factors in Computing Systems - CHI '13 (CHI '13). ACM Press, Paris, France, 189-192. https: //doi.org/10.1145/2470654.2470680

[41] Yiqin Lu, Chun Yu, Xin Yi, Yuanchun Shi, and Shengdong Zhao. 2017. BlindType Eyes-Free Text Entry on Handheld Touchpad by Leveraging Thumb's Muscle Memory. Proceedings of the ACM on Interactive, Mobile, Wearable and Ubiquitous Technologies 1, 2 (June 2017), 1-24. https://doi.org/10.1145/3090083

[42] Nicolai Marquardt, Ricardo Jota, Saul Greenberg, and Joaquim A. Jorge. 2011 The Continuous Interaction Space: Interaction Techniques Unifying Touch and Gesture on and above a Digital Surface. In Human-Computer Interaction - INTERACT 2011 (Lecture Notes in Computer Science), Pedro Campos, Nicholas Graham, Joaquim Jorge, Nuno Nunes, Philippe Palanque, and Marco Winckler (Eds.) Springer, Berlin, Heidelberg, 461-476. https://doi.org/10.1007/978-3-642-23765232

[43] Kim Marriott, Falk Schreiber, Tim Dwyer, Karsten Klein, Nathalie Henry Riche, Takayuki Itoh, Wolfgang Stuerzlinger, and Bruce H. Thomas (Eds.). 2018. Immer sive Analytics. Number 11190 in Lecture Notes in Computer Science. SpringerVerlag $\mathrm{GmbH}$, Cham.

[44] Alexandre Millette and Michael J. McGuffin. 2016. DualCAD: Integrating Augmented Reality with a Desktop GUI and Smartphone Interaction. In 2016 IEEE International Symposium on Mixed and Augmented Reality (ISMAR-Adjunct). IEEE, 21-26. https://doi.org/10.1109/ISMAR-Adjunct.2016.0030

[45] Tamara Munzner. 2014. Visualization Analysis and Design. A K Peters/CRC Press https://doi.org/10.1201/b17511

[46] Sharon Oviatt. 1999. Mutual Disambiguation of Recognition Errors in a Multimodel Architecture. In Proceedings of the SIGCHI conference on Human factors in computing systems the CHI is the limit - CHI '99. ACM Press, Pittsburgh, Pennsylvania, United States, 576-583. https://doi.org/10.1145/302979.303163

[47] Sharon Oviatt. 1999. Ten Myths of Multimodal Interaction. Commun. ACM 42 , 11 (nov 1999), 74-81. https://doi.org/10.1145/319382.319398

[48] Sharon Oviatt and Philip Cohen. 2000. Perceptual User Interfaces: Multimoda Interfaces That Process What Comes Naturally. Commun. ACM 43, 3 (mar 2000), 45-53. https://doi.org/10.1145/330534.330538

[49] Sharon Oviatt, Rebecca Lunsford, and Rachel Coulston. 2005. Individual Differences in Multimodal Integration Patterns: What Are They and Why Do They Exist?. In Proceedings of the SIGCHI conference on Human factors in computing systems - CHI '05 (Proceedings of the SIGCHI Conference on Human Factors in Computing Systems - CHI '05). ACM Press, New York, NY, USA, 241-249. Issue April. https://doi.org/10.1145/1054972.1055006

[50] Ken Pfeuffer, Yasmeen Abdrabou, Augusto Esteves, Radiah Rivu, Yomna Abdelrahman, Stefanie Meitner, Amr Saadi, and Florian Alt. 2021. ARtention: A Design Space for Gaze-Adaptive User Interfaces in Augmented Reality. Computers \& Graphics 95 (apr 2021), 1-12. https://doi.org/10.1016/j.cag.2021.01.001

[51] Ken Pfeuffer, Jason Alexander, Ming Ki Chong, and Hans Gellersen. 2014. GazeTouch: Combining Gaze with Multi-Touch for Interaction on the Same Surface. In Proceedings of the 27th Annual ACM Symposium on User Interface Software and Technology (UIST '14). ACM, New York, NY, USA, 509-518. https://doi.org/10. $1145 / 2642918.2647397$

[52] Ken Pfeuffer, Benedikt Mayer, Diako Mardanbegi, and Hans Gellersen. 2017. Gaze + Pinch Interaction in Virtual Reality. In Proceedings of the 5th Symposium on Spatial User Interaction (SUI '17). ACM, New York, NY, USA, 99-108. https: //doi.org/10.1145/3131277.3132180

[53] Peter J. Polack, Moushumi Sharmin, Kaya de Barbaro, Minsuk Kahng, Shang-Tse Chen, and Duen Horng Chau. 2017. Exploratory Visual Analytics of Mobile Health Data: Sensemaking Challenges and Opportunities. In Mobile Health: Sensors, Analytic Methods, and Applications, James M. Rehg, Susan A. Murphy, and Santosh Kumar (Eds.). Springer International Publishing, Cham, 349-360. https://doi.org/10.1007/978-3-319-51394-2 18

[54] Leah M. Reeves, Jean-Claude Martin, Michael McTear, Tv Raman, Kay M. Stanney, Hui Su, Qian Ying Wang, Jennifer Lai, James A. Larson, Sharon Oviatt, T. S. Balaji, Stéphanie Buisine, Penny Collings, Phil Cohen, and Ben Kraal. 2004. Guidelines for Multimodal User Interface Design. Commun. ACM 47, 1 (jan 2004), 57. https://doi.org/10.1145/962081.962106

[55] Carolin Reichherzer, Jack Fraser, Damien Constantine Rompapas, and Mark Billinghurst. 2021. SecondSight: A Framework for Cross-Device Augmented Reality Interfaces. In Extended Abstracts of the 2021 CHI Conference on Human Factors in Computing Systems (CHI EA '21). ACM, New York, NY, USA, 1-6. https://doi.org/10.1145/3411763.3451839

[56] Patrick Reipschläger and Raimund Dachselt. 2019. DesignAR: Immersive 3DModeling Combining Augmented Reality with Interactive Displays. In Proceedings of the 2019 ACM International Conference on Interactive Surfaces and Spaces. ACM Press, Daejeon, Republic of Korea, 29-41. https://doi.org/10.1145/3343055. 3359718

[57] Patrick Reipschläger, Tamara Flemisch, and Raimund Dachselt. 2021. Personal Augmented Reality for Information Visualization on Large Interactive Displays. IEEE Transactions on Visualization and Computer Graphics 27, 2 (feb 2021), 11821192. https://doi.org/10.1109/TVCG.2020.3030460 arXiv:2009.03237

[58] Donghao Ren, Bongshin Lee, and Tobias Höllerer. 2018. XRCreator: Interactive Construction of Immersive Data-Driven Stories. In Proceedings of the 24th ACM Symposium on Virtual Reality Software and Technology (VRST '18). ACM, New York, NY, USA, 1-2. https://doi.org/10.1145/3281505.3283400

[59] Gang Ren and Eamonn O'Neill. 2013. 3D Selection with Freehand Gesture. Computers \& Graphics 37, 3 (may 2013), 101-120. https://doi.org/10.1016/j.cag. 2012.12.006

[60] Radiah Rivu, Yasmeen Abdrabou, Ken Pfeuffer, Augusto Esteves, Stefanie Meitner, and Florian Alt. 2020. StARe: Gaze-Assisted Face-to-Face Communication in Augmented Reality. In ACM Symposium on Eye Tracking Research and Applications (ETRA '20 Adjunct). ACM, New York, NY, USA, 1-5. https://doi.org/10.1145/ 3379157.3388930

[61] Ayshwarya Saktheeswaran, Arjun Srinivasan, and John Stasko. 2020. Touch? Speech? Or Touch and Speech? Investigating Multimodal Interaction for Visual Network Exploration and Analysis. IEEE Transactions on Visualization and Computer Graphics 26, 6 (jun 2020), 2168-2179. https://doi.org/10.1109/TVCG.2020. 2970512

[62] Marc Satkowski and Raimund Dachselt. 2021. Investigating the Impact of RealWorld Environments on the Perception of 2D Visualizations in Augmented Reality. In Proceedings of the 2021 CHI Conference on Human Factors in Computing Systems. ACM, New York, NY, USA, 1-15. https://doi.org/10.1145/3411764.3445330

[63] Ben Shneiderman. 1996. The Eyes Have It: A Task by Data Type Taxonomy for Information Visualizations. In Proceedings 1996 IEEE Symposium on Visual Languages. IEEE Comput. Soc. Press, Boulder, CO, USA, 336-343. https://doi. org/10.1109/VL.1996.545307

[64] Anthony Smith, Kristy de Salas, Benjamin Schüz, Stuart G Ferguson, and Ian Lewis. 2016. mHealth Intervention Design: Creating mHealth Interventions for Behaviour Change. In Proceedings of the 28th Australian Conference on ComputerHuman Interaction - $\mathrm{OzCHI}$ '16 (OzCHI '16). ACM Press, Launceston, Tasmania, Australia, 531-536. https://doi.org/10.1145/3010915.3010986

[65] Arjun Srinivasan, Bongshin Lee, Nathalie Henry Riche, Steven M. Drucker, and Ken Hinckley. 2020. InChorus: Designing Consistent Multimodal Interactions for Data Visualization on Tablet Devices. In Proceedings of the 2020 CHI Conference on Human Factors in Computing Systems (CHI '20). Association for Computing Machinery, Honolulu, HI, USA, 1-13. https://doi.org/10.1145/3313831.3376782

[66] Arjun Srinivasan and John Stasko. 2018. Orko: Facilitating Multimodal Interaction for Visual Exploration and Analysis of Networks. IEEE Transactions on Visualization and Computer Graphics 24, 1 (jan 2018), 511-521. https: //doi.org/10.1109/TVCG.2017.2745219

[67] Jennifer G. Stadler, Kipp Donlon, Jordan D. Siewert, Tessa Franken, and Nathaniel E. Lewis. 2016. Improving the Efficiency and Ease of Healthcare Analysis Through Use of Data Visualization Dashboards. Big Data 4, 2 (jun 2016), 129-135. https://doi.org/10.1089/big.2015.0059

[68] Hemant Bhaskar Surale, Aakar Gupta, Mark Hancock, and Daniel Vogel. 2019. TabletInVR: Exploring the Design Space for Using a Multi-Touch Tablet in Virtual Reality. In Proceedings of the 2019 CHI Conference on Human Factors in Computing Systems - CHI '19. ACM Press, Glasgow, Scotland Uk, 1-13. https://doi.org/10. $1145 / 3290605.3300243$

[69] Slawomir Konrad Tadeja, Timoleon Kipouros, and Per Ola Kristensson. 2019. Exploring Parallel Coordinates Plots in Virtual Reality. In Extended Abstracts of the 2019 CHI Conference on Human Factors in Computing Systems - CHI EA '19. ACM Press, Glasgow, Scotland Uk, 1-6. https://doi.org/10.1145/3290607.3313068 
[70] Philipp Wacker, Oliver Nowak, Simon Voelker, and Jan Borchers. 2019. ARPen: Mid-Air Object Manipulation Techniques for a Bimanual AR System with Pen \& Smartphone. In Proceedings of the 2019 CHI Conference on Human Factors in Computing Systems - CHI '19 (CHI '19). ACM Press, Glasgow, Scotland Uk, 1-12. https://doi.org/10.1145/3290605.3300849

[71] Dirk Wenig, Johannes Schöning, Alex Olwal, Mathias Oben, and Rainer Malaka 2017. WatchThru: Expanding Smartwatch Displays with Mid-Air Visuals and Wrist-Worn Augmented Reality. In Proceedings of the 2017 CHI Conference on Human Factors in Computing Systems (CHI '17). ACM, Denver, Colorado, USA, 716-721. https://doi.org/10.1145/3025453.3025852

[72] R. Wilton and A. J. Pennisi. 1993. Evaluating the Accuracy of Transcribed Clinical Data. Proceedings of the Annual Symposium on Computer Application in Medical Care (1993), 279-283.

[73] Dennis Wolf, Jan Gugenheimer, Marco Combosch, and Enrico Rukzio. 2020 Understanding the Heisenberg Effect of Spatial Interaction: A Selection Induced Error for Spatially Tracked Input Devices. In Proceedings of the 2020 CHI Conference on Human Factors in Computing Systems (Honolulu, HI, USA) (CHI '20). Association for Computing Machinery, New York, NY, USA, 1-10. https://doi.org/10.1145/3313831.3376876
[74] Wanze Xie, Yining Liang, Janet Johnson, Andrea Mower, Samuel Burns, Colleen Chelini, Paul D. Alessandro, Nadir Weibel, and Jürgen P. Schulze. 2020. Interactive Multi-User 3D Visual Analytics in Augmented Reality. arXiv:2002.05305 [cs] (Feb. 2020). arXiv:2002.05305 [cs]

[75] Johannes Zagermann, Ulrike Pfeil, Philipp von Bauer, Daniel Fink, and Harald Reiterer. 2020. "It's in My Other Hand!" - Studying the Interplay of Interaction Techniques and Multi-Tablet Activities. In Proceedings of the 2020 CHI Conference on Human Factors in Computing Systems (Honolulu, HI, USA) (CHI '20). Association for Computing Machinery, New York, NY, USA, 1-13. https://doi.org/10.1145/3313831.3376540

[76] Fengyuan Zhu and Tovi Grossman. 2020. BISHARE: Exploring Bidirectional Interactions Between Smartphones and Head-Mounted Augmented Reality. In Proceedings of the 2020 CHI Conference on Human Factors in Computing Systems (CHI '20). Association for Computing Machinery, Honolulu, HI, USA, 1-14. https: //doi.org/10.1145/3313831.3376233

[77] P. Ziuziański, M. Furmankiewicz, and A. Sołtysik-Piorunkiewicz. 2014. E-Health Artificial Intelligence System Implementation: Case Study of Knowledge Management Dashboard of Epidemiological Data in Poland. International fournal of Biology and Biomedical Engineering 8 (2014), 8. 